\title{
Absent Pulmonary Valve Syndrome with Tetralogy of Fallot and Patent Ductus Arteriosus at 14 Weeks of Gestation and Follow-up 2 weeks later: Case Report and Review of Literature
}

\author{
Yan Song ${ }^{1}$, Yu-fen Zou ${ }^{1}$, Yan-hui Ru${ }^{1}$, Jing Qiu ${ }^{1}$, and Hong Yin ${ }^{1}$ \\ ${ }^{1}$ Affiliation not available
}

June 28, 2020

\begin{abstract}
Classical absent pulmonary valve syndrome (APVS) with tetralogy of Fallot (TOF) is a rare congenital cardiac anomaly commonly associated with absent of patent ductus arteriosus (PDA), which is mostly diagnosed after 20 weeks of gestation by fetal echocardiography. This case of APVS with TOF was suspected at 13 weeks of gestation and diagnosed at 14 weeks of gestation with an obvious PDA. The pulmonary arterial trunk and the branches did not dilated obviously. 15 days later, the PDA narrowed down with the discovery of pulmonary artery ectasia at the same time. This progress indicated that the absent of PDA is not necessary for the survival of APVS with TOF in utero, in contrast, the absent or restriction of PDA may be nothing less than adaptation to the disease. Fetal autopsy confirmed the accuracy of fetal echocardiography. Chromosome microarray analysis (CMA) showed 20p12 deletion in this fetus, which is rare among TOF cases.
\end{abstract}

\section{Absent Pulmonary Valve Syndrome with Tetralogy of Fallot and Patent Ductus Arteriosus at 14 Weeks of Gestation and Follow-up 2 weeks later: Case Report and Review of Literature}

Yan Song, Yu-fen Zou, Yan-hui Ru, Jing Qiu, Hong Yin ${ }^{1}$.

Department of Ultrasound, Shandong Provincial Maternal and Child Health Hospital, Jinan, China

1, Corresponding Author

Classical absent pulmonary valve syndrome (APVS) with tetralogy of Fallot (TOF) is a rare congenital cardiac anomaly commonly associated with absent of patent ductus arteriosus (PDA), which is mostly diagnosed after 20 weeks of gestation by fetal echocardiography. This case of APVS with TOF was suspected at 13 weeks of gestation and diagnosed at 14 weeks of gestation with an obvious PDA. The pulmonary arterial trunk and the branches did not dilated obviously. 15 days later, the PDA narrowed down with the discovery of pulmonary artery ectasia at the same time. This progress indicated that the absent of PDA is not necessary for the survival of APVS with TOF in utero, in contrast, the absent or restriction of PDA may be nothing less than adaptation to the disease. Fetal autopsy confirmed the accuracy of fetal echocardiography. Chromosome microarray analysis (CMA) showed 20p12 deletion in this fetus, which is rare among TOF cases.

KEYWORDS congenital heart disease, fetal echocardiography, pulmonary valve, Tetralogy of Fallot

\section{1. case report}

At the outset, a 36-year-old pregnant woman without consanguineous history was suspected absent pulmonary valve syndrome (APVS) in fetus in the first trimester screening in our Hospital. The pregnancy is natural with no history of fever, discomfort, allergies and medication in early period. The biological 
parameters were in accordance with those of 13th gestational weeks. Fetal nuchal translucency (NT) was in normal range $(0.19 \mathrm{~cm})$, the pulsed doppler spectrum of ductus venosus (DV) was normal in shape. The blood flow through mitral valve and tricuspid valve in the 4-chamber view was symmetrical. However, in the 3-vessel tracheal view, there were anterograde blood flows in systole and retrograde blood flow in diastole in pulmonary artery (PA) and patent ductus arteriosus (PDA), which suggested absent of pulmonary valve. Due to the obesity and rejection of transvaginal examination of the patient, a meticulous fetal heart examination was arranged 7 days later and more positive results were found: bidirectional blood flow in the upper segment of interventricular septum (beam width $0.14 \mathrm{~cm}$ ); overriding aorta; narrowed pulmonary valve annulus with pulmonary valve unexplored; moderate reflux flow of tricuspid valve. To-and-from blood flow was detected clearly through pulmonary annulus, PA trunk, left pulmonary artery (LPA), right pulmonary artery (RPA) and PDA. By these features, APVS with tetralogy of Fallot (TOF) was diagnosed (Figure 1). The shape and size of PA trunk, PA branches and PDA still seemed normal.

The fetus was reexamined 15 days later. The biparietal diameter (BPD) was $3.2 \mathrm{~cm}$ and femoral diameter (FL) was $1.9 \mathrm{~cm}$, which were consistent with 16 weeks of pregnancy. The right heart was slightly larger than left heart in 4-chamber view, the width of ventricular septal defect (VSD) was $0.17 \mathrm{~cm}$. The diameter of aortic annulus riding over the VSD was $0.24 \mathrm{~cm}$. Pulmonary valve leaflets were undetectable during dynamic observation. The diameters of pulmonary valve annulus, pulmonary trunk, LPA, RPA, DA were clearly measured as $0.16 \mathrm{~cm}, 0.23 \mathrm{~cm}, 0.19 \mathrm{~cm}, 0.20 \mathrm{~cm}, 0.13 \mathrm{~cm}$ respectively. The ectasia of pulmonary trunk and branches combined with the stenosis of annulus constituted a distinct "goldfish tail sign". Despite of the to-and-fro flow, the blood flow mosaic was detected in pulmonary trunk which proved the acceleration of flow through pulmonary valve annulus. DA was much narrower than pulmonary trunk and branches with comparatively dim blood flow through, suggesting the restriction of DA with decreased blood flow (Figure 2 ). Except for fetal cardiac anomalies, left renal cyst $(0.4 \times 0.4 \mathrm{~cm})$ was found in fetus with normal amniotic fluid.

Approved by the ethics committee of our hospital, fetal induction was performed. Fetal autopsy approved by the pregnant mother confirmed the diagnosis of APVS with TOF by fetal echocardiography (Figure 3). Chromosome microarray chip analysis (CMA) showed that the $984.766 \mathrm{~kb}$ fragment at 20p12.2 deleted.

\section{DISCUSSION}

APVS is a rare congenital heart disease (CHD) with an incidence of $0.2 \%-0.4 \%$ in all live birth with CHD, characterized by absent or severe dysplasia of pulmonary valve leaflets ${ }^{[1-3]}$. APVS is commonly classified into two types: The majority of APVS fetuses is accompanied with TOF, so defined as TOF-type APVS; the minority is absent from VSD, defined as non-TOF-type APVS ${ }^{[3,4]}$. TOF-type APVS usually got stenosis of pulmonary valve annulus, post-narrow expansion of PA and absent PDA in the previous studies. The inflation of PA trunk and RPA may compress the airway to collapse and softening, which leads to respiratory distress and heart failure in neonatal period. The clinical manifestations are critical, coupled with extracardiac malformations and / or chromosomal abnormalities, the overall prognosis is poor. Non-TOF-type APVS is commonly associated with intact ventricular septum, tricuspid valve atresia or hypoplasia and PDA. The PA trunk usually does not expand, but shows hypoplasia, which proceeds to the immaturity of vessel and tissues in lung. The total mortality of APVS was 75\%, including intrauterine death and postnatal death, only $25 \%$ were alive after postnatal surgery; therefore, prenatal diagnosis of APVS is significant ${ }^{[3]}$. Although the postoperative survival rate has improved in recent years, the heart surgery is

still complicated. Sometimes heart transplantation is required in emergency ${ }^{[5]}$. So early diagnosis of APVS in utero is meaningful.

Most TOF-type APVS fetuses are diagnosed after 20 weeks of gestation by echocardiography. Except for positive grayscale images, Color doppler flow imaging (CDFI) shows color mosaic to-and-from blood flow in PA. Spectrum Doppler shows high-speed blood flow antegrade and retrograde in PA. In this study, based on the high resolution of ultrasound, APVS was suspected at 13 weeks of gestation by a skilled sonographer in the first trimester screening, and was confirmed 7 days later. Two weeks later, fetal echocardiography was performed again. In the last two checks, PA and PDA were found with obvious morphological change, which 
might be helpful in the study of mechanism and development of TOF-type APVS in early middle pregnancy.

In the past, absence of PDA has always been considered in company with dilation of PA in fetus with TOF-type APVS, because the insufficient pulmonary systemic shunt aggravated volume load of RV caused by to-and-from blood flow in PA ${ }^{[6,7]}$. However, such claims are practically based on the discoveries of TOF-type APVS after 20 weeks of gestation. The process and pathogenesis before 20 weeks were unclear with controversies in correlation between PA dilatation and PDA absence. In the present case at 14 weeks of gestation, the diameter of PDA was wide with unobstructed bidirectional shunt. PA expansion was not distinct. At 16 weeks of gestation, there was PA expansion without PDA absence, which was obviously inconsistent with previous study. The restriction of PDA might be associated with the dilation of PA trunk and PA branches, which leaded to the decrease of blood flow volume through PDA. It advocated the controversial hypothesis that APVS with TOF leads to depressed diastolic pressure of immature PDA and ultimately result in the restriction or closure at an early gestational age ${ }^{[8]}$. It suggested the dynamic pathological change of PDA in TOF-type APVS fetus in early middle pregnancy. If the fetus developed further in utero, disuse atrophy or atresia of PDA might occur in the future. In addition, it has been reported that several fetuses with TOF-type APVS had constricted PDA after 20 weeks of gestation. It also confirmed that the absence of PDA is not inevitable for the existence of TOF-type APVS. It further proves the rationality of the inference in this study.

APVS may be associated with fetal chromosomal abnormalities, among which 22q11 microdeletions, 13 trisomy and 18 trisomy are most common ${ }^{[9]}$. It is suggested that $70 \%$ of TOF-type APVS were associated with karyotype abnormality. In this case, $984.766 \mathrm{~kb}$ deletion was detected at 20p12.2 by chromosome microarray analysis (CMA). JAG1 gene in this fragment is in autosomal dominant inheritance, and the deletion is related to TOF and Alagille syndrome. The parents of the fetus were in good health without genetic defects and the deletion of JAG1 gene in fetus was thought to be caused by gene mutation and lead to poor prognosis.

TOF-type APVS is combined with cardiac extracardiac malformations sometimes which is independent influencing factor of prognosis. In this case, a left renal cyst was found which might be sign of cystic dysplasia of kidney.

In this case, the pregnancy was terminated. Fetal autopsy confirmed the ultrasound diagnosis.

\section{CONCLUSION}

This case of TOF-type APVS with was diagnosed at 14 weeks of gestation with an obvious PDA. 15 days later, the PDA narrowed down together with ectasia of PA. This progress indicated that the absent of PDA is not necessary for the survival of TOF-type APVS in utero, in contrast, the absent or restriction of PDA may be nothing less than adaptation to the disease. 20p12 deletion was found in this fetus. Prenatal diagnosis in early stage is conducive to reasonable clinical decision-making.

\section{REFERENCES}

1. Allan LD, Sharland GK, Milburn A, et al. Prospective diagnosis of 1,006 consecutive cases of congenital heart disease in the fetus. J Am Coll Cardiol. 1994;23(6):1452-58

2. Szwast A, Tian Z, McCann M, et al. Anatomic variability and outcome in prenatally diagnosed absent pulmonary valve syndrome. Ann Thorac Surg. 2014;98(1):152-158.

3. Volpe P, Paladini D, Marasini M, et al. Characteristics, associations and outcome of absent pulmonary valve syndrome in the fetus. Ultrasound Obstet Gynecol 2004;24:623-8

4. Zucker N, Rozin I, Levitas A, et al. Clinical presentation, natural history, and outcome of patients with the absent pulmonary valve syndrome. Cardiol Young. 2004;14(4):402-8.

5. Razavi RS, Sharland GK, Simpson JM. Prenatal diagnosis by echocardiogram and outcome of absent pulmonary valve syndrome. [J] Am J Cardiol 2003,91( 4): 429-432

6. Wertaschnigg D, Jaeggi M, Chitayat D, et al Prenatal diagnosis and outcome of absent pulmonary valve syndrome: contemporary single-center experience and review of the literature $\mathrm{J}$ Ultrasound 
Obstet Gynecol 2013, 41( 2): 162-167

7. Joshi AN, Rane HS, Kamble RC, et al. Prenatal diagnosis of absent pulmonary valve syndrome: report of 2 cases, most common and most rare presentations [ J]. J Ultrasound Med 2010,29(5): 823-829

8. Becker R, Schmitz L, Guschmann M, et al. Prenatal diagnosis of familial absent pulmonary valve syndrome: case report and review of the literature. Ultrasound Obstet Gynecol. 2001; 17(3):263-7

9. Sleurs E, De Catte L,Benatar A. Prenatal diagnosis of absent pulmonary valve syndrome in association with 22q11 deletion [J]. J Ultrasound Med 2004233 417-422
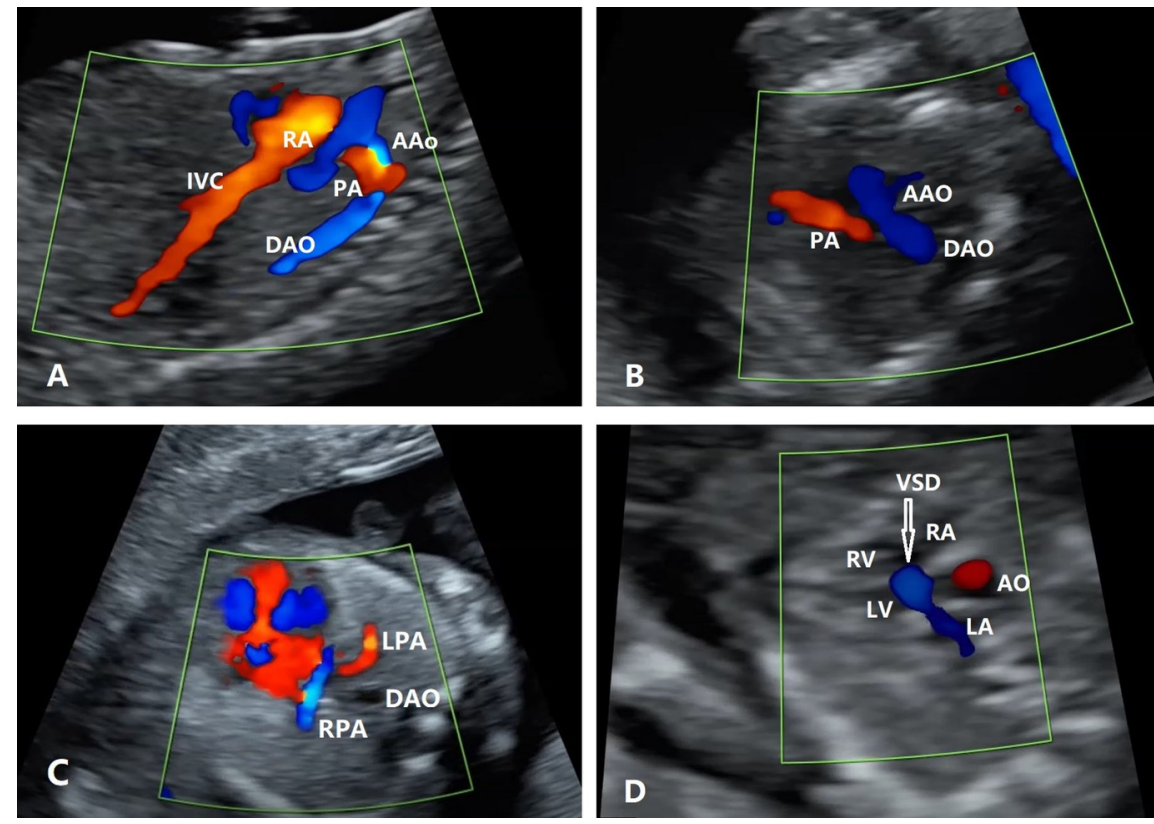

Figure 1 Echocardiography of APVS at 14 weeks of gestation

A. Longitudinal view of DA showed the retrograde blood flow from DA to PA; B. 3VT view showed the retrograde blood flow in PA; C. Oblique PA branches view showed that RPA and LPA were not dilated and the blood flow was unobstructed; D. 4 chamber view showed poorly aligned VSD

VSD: ventricular septal defect; AAO: aortic arch; DAO: descending aorta; PA: pulmonary artery; LPA: left pulmonary artery; RPA: right pulmonary artery; RV: right ventricle; LV: left ventricle; RA: right atrium; LA: left atrium; IVC: inferior vena cava; VSD: ventricular septal defect

White arrow indicates right to left shunt through VSD 

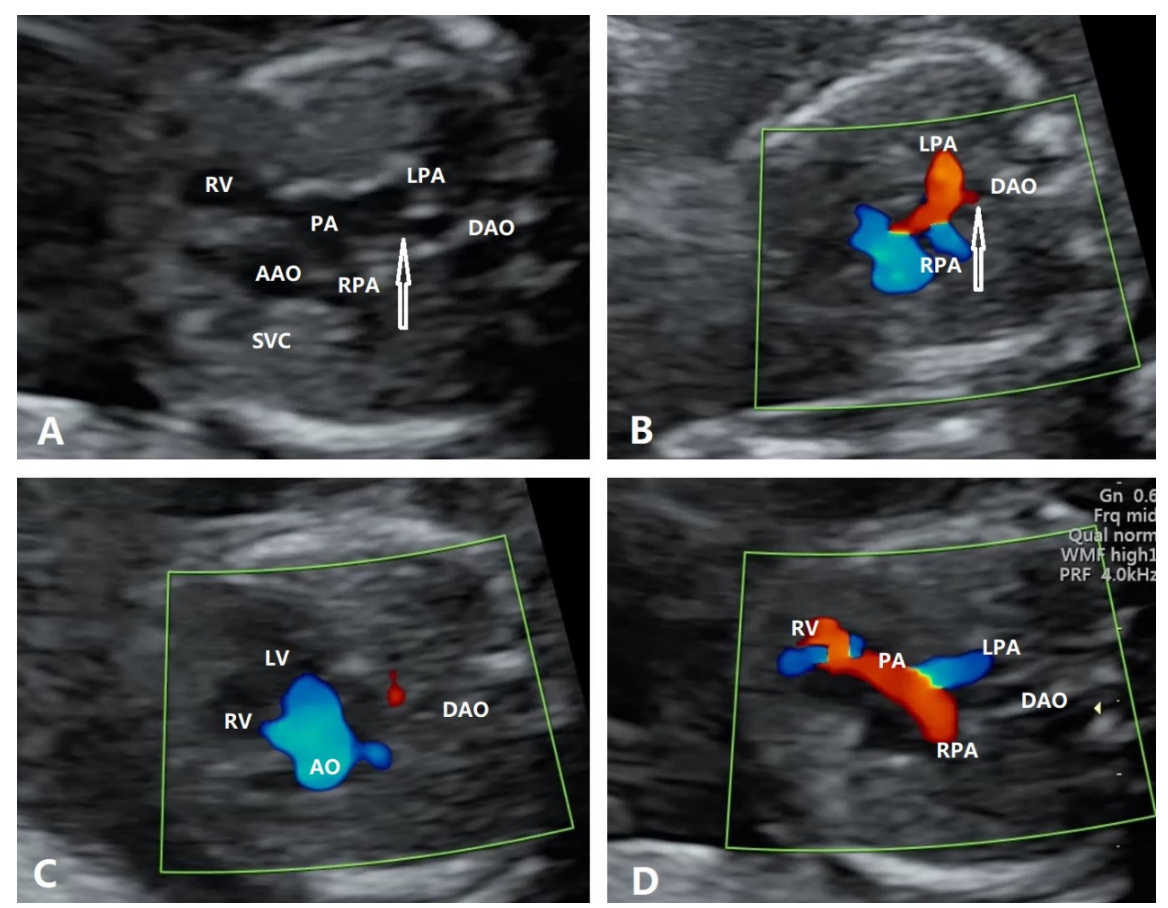

Figure 2 Echocardiography of APVS at 16 weeks of gestation

A. 2D nonstandard axis view of DA showed the narrowed pulmonary annulus, expansive pulmonary trunk and branches and narrowed DA ; B. CDFI showed the wide and bright antegrade blood flow in PA trunk and branches, and the dark blood flow in DA from PA to DAO; C. 5 chamber view showed the blood flow from RV and LV to the overriding AO; D. PA longitudinal view showed the retrograde blood flow from LPA and RPA to PA trunk and RV.

AAO: aortic arch; DAO: descending aorta; PA: pulmonary artery; LPA: left pulmonary artery; RPA: right pulmonary artery; RV: right ventricle; LV: left ventricle; AO: aortic artery.

White arrow showed DA 

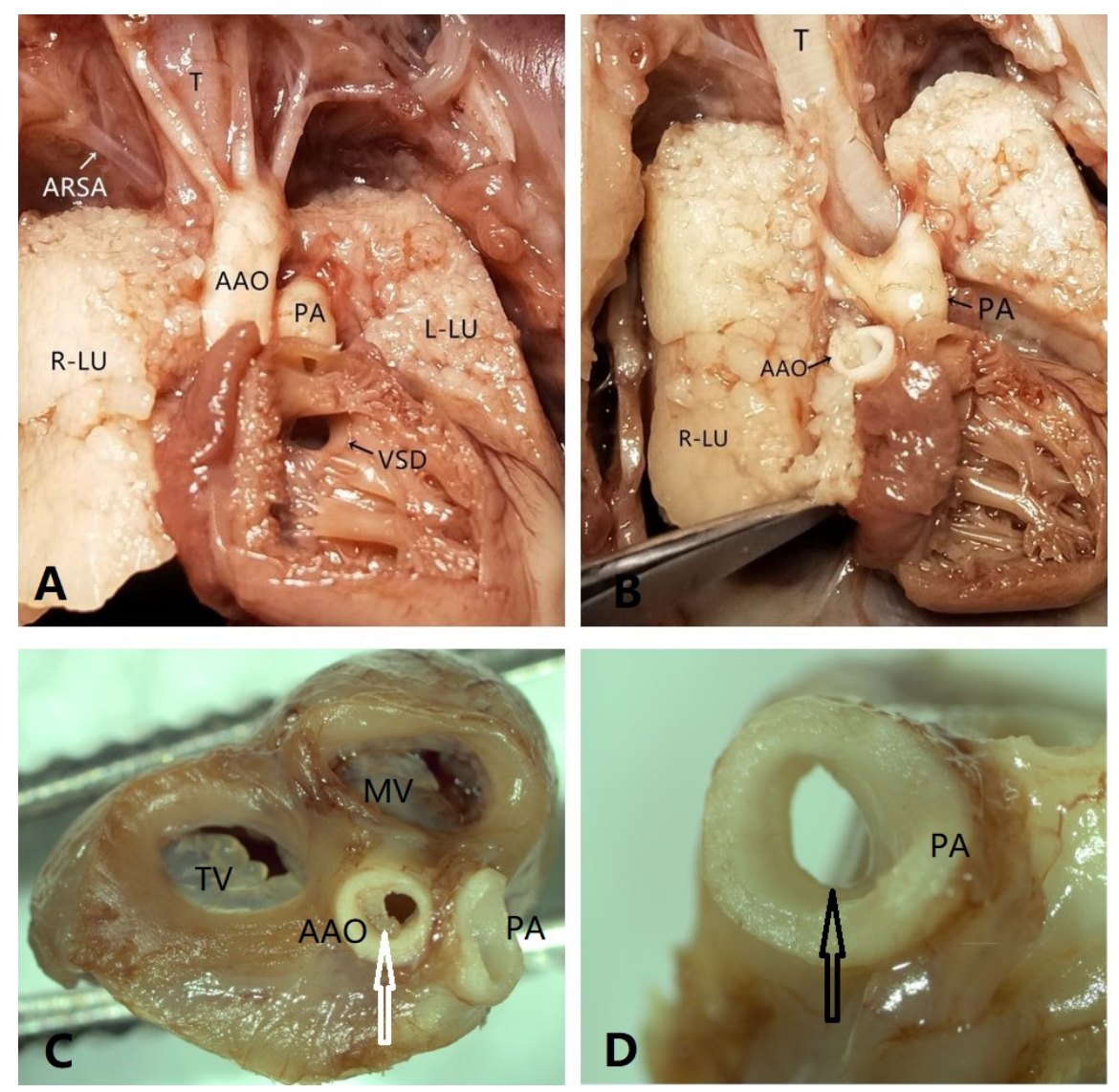

Figure 3 Anatomical images of APVS heart at 16 weeks of gestation

A. The front view of fetal heart removing the right ventricular wall showed the upper VSD and ARSA ; B. PA view showed the expanded PA trunk and branches; C. The upper view of TV, MV and AV, all the three valves were clearly visible; $\mathrm{D}$. The upper view of pulmonary annulus, there was no relic of PV in the pulmonary annulus.

VSD: ventricular septal defect; AAO: aortic arch; PA: pulmonary artery; VSD: ventricular septal defect; ARSA: the aberrant right subclavian artery; R-LU: right lung; L-LU: left lung; T: trachea; TV: tricuspid valve; MV: mitral valve.

White arrow showed the aortic valve leaflets. Black arrow showed the pulmonary annulus without pulmonary valve leaflet. 